\title{
Factors which influence the timing of maximum growth rate of the head in low birthweight infants
}

\author{
MASANORI FUJIMURA \\ From the Department of Paediatrics, University of Oxford, John Radcliffe Hospital, Oxford
}

SUMMARY The postnatal rate of head growth in 63 infants of less than 34 weeks' gestation was examined retrospectively, together with that of 7 other infants of between 34 and 40 weeks' gestation who had severe respiratory illnesses. From the serial measurements of head circumference the timing of maximum velocity of postnatal head growth was examined and related to the infant's gestational age, nutrition, and respiratory illness during the neonatal period. Within this group of infants the longer the gestation the earlier maximum head growth velocity occurred. For infants of similar gestational ages the presence of a severe respiratory problem was associated with a delay in the time of maximum head growth. Differences in caloric intake during the first week of life did not seem to affect significantly the timing of maximum head growth velocity.

The human brain grows most rapidly during the perinatal period (Dobbing and Sands, 1973). It has been well documented that the increase in occipitofrontal head circumference (OFC) correlates well with the growth of the intracranial volume and of the brain during infancy and childhood (Bray et al., 1969; Winick and Rosso, 1969; Buda et al., 1975).

Although charts of OFC growth of term infants are available, this is not so for preterm infants. Among the few studies of head growth of preterm infants, O'Neill's (1961) is one of the earliest. Until recently the head growth of preterm infants has not been clearly differentiated from that of term infants (Sher and Brown, 1975a).

Variations in food intake and body temperature of the preterm infant have been reported to affect the speed of head growth (Davies and Davis, 1970; Glass et al., 1975). Infants with the idiopathic respiratory distress syndrome and/or those who required prolonged intravenous feeding have shown retarded head growth during the neonatal period (Sher and Brown, 1975b).

On the other hand, my studies have shown differences in velocity curves of OFC growth in term and preterm infants who were otherwise appropriate weight for dates (AFD) (Fujimura and Seryu, 1977). Term AFD infants showed continuously decreasing growth velocity of OFC with increasing postnatal age. Preterm AFD infants of less than 36 weeks' gestation had an initial increase in growth velocity followed by a decrease, with the maximum velocity

Received 15 May 1976 occurring between 30 and 40 days after birth. The smaller the infant the later maximum velocity occurred. Different patterns of postnatal head growth may be conveniently examined by looking at the postnatal age at which maximum head growth velocity occurs.

This study examines the effects of gestational age at birth, postnatal nutrition, and respiratory illness on the timing of maximum head growth velocity.

\section{Patients and methods}

The infants were cared for in the Special Care Baby Unit, John Radcliffe Hospital, between 1972 and 1973. All preterm or sick newborns were first fed 2 to 3 hours after birth unless intravenous glucose administration was indicated (Davies et al., 1972). Environmental temperature was controlled according to the guidelines proposed by Hey and Katz (1970). Excluding infants who died, those who were of multiple pregnancy, and those with congenital abnormality or cranial pathology, 63 infants of less than 34 completed weeks of gestation were studied, together with 7 others of $34-40$ weeks who required oxygen therapy for more than 7 days postnatally. None has developed any subsequent neurological defect. During the stay in the Special Care Baby Unit, OFC was measured by the staff once a week using disposable paper tape measures. Body weight was measured three times a week on a balance calibrated to $10 \mathrm{~g}$. Some of the information was obtained from the observations made in the outpatient clinic. 


\section{Fujimura}

Fig. 1 shows how the speed of head growth was examined and how the timing of maximum head growth was calculated in each case. Taking the nearest 2 points of OFC separated by 25 days or more, the increment of OFC was divided by the number of days, thus giving the mean daily velocity during this period. From the velocity curve of OFC shown on the lower half of Fig. 1, the timing of highest mean velocity of head growth was obtained. Variability in the measurement of OFC is diminished because the increment is divided by a large number of days. Mean velocity values before the age of 12 days cannot be calculated with this method. If the maximum velocity appears to be the first point on the curve, then the real timing of maximum velocity may have been earlier than this first point.

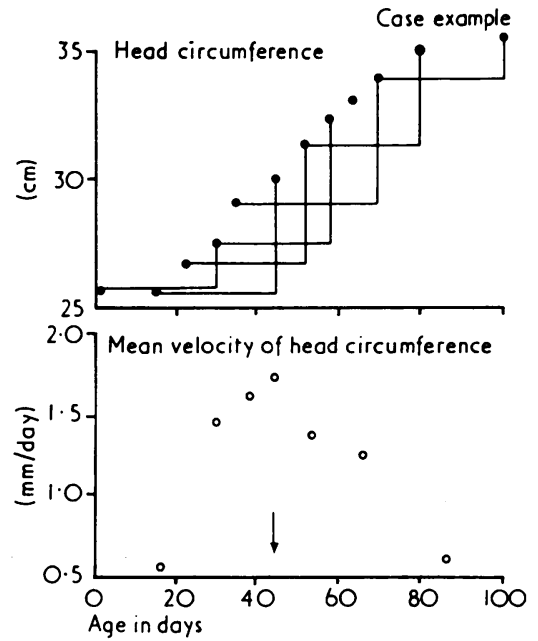

Fig. 1 A case example showing how the timing of maximum head growth was obtained. Taking the nearest 2 points of OFC (occipitofrontal head circumference) separated by 25 days or more (upper half), the increment of $O F C$ was divided by the number of days, and the mean velocity during the period was obtained (lower half).
Postnatal change of the shape of skull in low birthweight infants has been considered as a possible source of error for the estimation of velocity. The difference between real and corrected OFC during the first year was reported to be approximately $10 \mathrm{~mm}$ at the most (Baum and Searls, 1971), which is in the order of $10^{-2} \mathrm{~mm}$ for the daily velocity value and can be neglected for the purposes of this study. Student's ' $t$ ' test was used for assessment of significance of difference in group comparisons.

\section{Results}

Infants of less than 34 weeks' gestation were divided into three groups according to the length of oxygen therapy after birth (Table 1). Infants in group $\mathbf{A}$ had at most transient respiratory distress. In groups $\mathbf{B}$ and $\mathbf{C}$ the proportion with respiratory distress, as diagnosed by accepted criteria (Davies et al., 1972), was $94 \%$ and $90 \%$, respectively. The proportion of infants requiring assisted ventilation was larger in group $\mathbf{C}$, indicating the severity of respiratory illness in this group. The groups differed in gestational age, birthweight, and OFC at birth but were similar in sex ratio. Group $D$ will be discussed at the end of the results.

Taking groups $\mathrm{A}, \mathrm{B}$, and $\mathrm{C}$ as a whole there was no correlation between the absolute growth of OFC during the first month of life and the gestational age at birth (Fig. 2, $r=0.01$ ). The timing of maximum velocity of head growth in the three groups of infants is given in Table 2 . It was significantly earlier in group $A$ than in groups $B$ and $C(P<0.001)$. A significant inverse correlation was found between the timing of maximum velocity of head growth and gestational age (Fig. 3, $\mathrm{r}=0 \cdot 50, \mathrm{P}<0.01$ ). Excluding the infants of less than 29 weeks' gestation, the gestational ages of the three groups were not different, whereas significant differences of the timing of maximum velocity were shown between groups $\mathrm{A}-\mathrm{B}$ and $\mathrm{A}-\mathrm{C}$ (Table 3 ).

Table 1 Details of infants studied

\begin{tabular}{|c|c|c|c|c|}
\hline Group & $\boldsymbol{A}$ & $B$ & $C$ & $D$ \\
\hline $\begin{array}{l}\text { Length of } \mathrm{O}_{2} \text { therapy (d) } \\
\text { No. of cases } \\
\text { Male/female } \\
\text { No. with respiratory distress } \\
\text { CPAP } \\
\text { IPPV } \\
\text { Gestational age (w) (mean } \pm \text { SD) } \\
\text { Birthweight }(\mathrm{g})(\text { mean } \pm \text { SD) } \\
\text { Head circumference at birth (cm) } \\
\text { (mean } \pm \text { SD) }\end{array}$ & $\begin{array}{l}<1 \\
25 \\
15 / 10 \\
9 \\
0 \\
0 \\
32 \cdot 0 \pm 1 \cdot 2 \\
1775 \pm 419 \\
30 \cdot 0 \pm 1 \cdot 7\end{array}$ & $\begin{array}{l}1-6 \\
18 \\
10 / 8 \\
17 \\
4 \\
1 \\
31 \cdot 5 \pm 1 \cdot 6 \\
1533 \pm 328 \dagger\end{array}$ & $\begin{array}{l}\geq 7 \\
20 \\
12 / 8 \\
18 \\
13 \\
10 \\
29 \cdot 3 \pm 1 \cdot 9 * \\
1394 \pm 347 \dagger \\
27 \cdot 8 \pm 2 \cdot 1 \dagger\end{array}$ & $\begin{array}{l}\geq 7 \\
7 \\
2 / 6 \\
7 \\
7 \\
7 \\
36 \cdot 5 \pm 1 \cdot 9 \\
2481 \pm 179 \\
33 \cdot 1 \pm 1 \cdot 1\end{array}$ \\
\hline
\end{tabular}

*Significantly different from groups A and B.

†Significantly different from group $A$.

CPAP = continuous positive airway pressure; IPPV =intermittent positive pressure ventilation. 


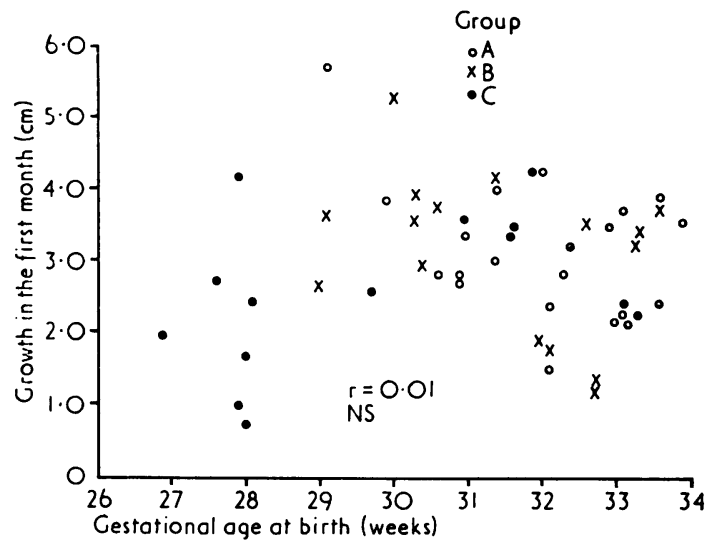

Fig. 2 Absolute growth of OFC plotted against gestational age at birth. No significant correlation was obtained.

Table 2 Timing of maximum velocity of $O F C$

\begin{tabular}{ll}
\hline Group & $\begin{array}{l}\text { Day of maximum velocity } \\
(\text { mean } \pm S D)\end{array}$ \\
\hline A & $19 \cdot 5 \pm 5 \cdot 8$ \\
B & $29 \cdot 9 \pm 8 \cdot 7^{*}$ \\
D & $35 \cdot 4 \pm 9 \cdot 9^{*}$ \\
\hline
\end{tabular}

*Significantly different from group A: P $<0.001$.

Table 3 Gestational ages and timing of maximum velocity of groups $A, B$, and $C$ with gestational ages $\geq 29$ weeks

\begin{tabular}{lll}
\hline & $\begin{array}{l}\text { Gestational age } \\
(w)(\text { mean } \pm S D)\end{array}$ & $\begin{array}{l}\text { Day of maximum } \\
\text { velocity } \\
(\text { mean } \pm S D)\end{array}$ \\
\hline $\mathrm{A}$ & $32 \cdot 0 \pm 1 \cdot 2$ & $19 \cdot 5 \pm 5 \cdot 8$ \\
$\mathrm{C}$ & $31 \cdot 5 \pm 1 \cdot 6$ & $29 \cdot 9 \pm 8 \cdot 7^{*}$ \\
\hline
\end{tabular}

*Significantly different from group A: P $<0.001$.

The effect of caloric intake on the timing of maximum velocity was also examined in infants of less than 34 weeks of gestation. During the first week of life the caloric intake of group $\mathrm{C}$ was significantly lower than that of groups A and B (Table 4,

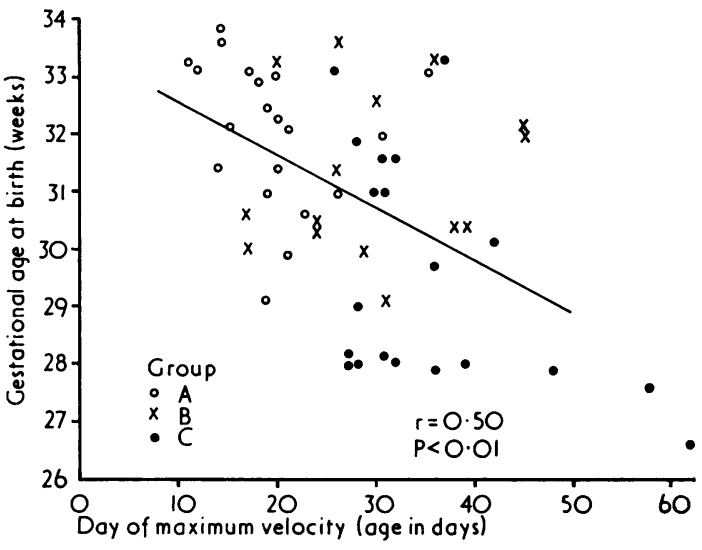

Fig. 3 Timing of maximum velocity of OFC growth plotted against gestational age at birth.

$P<0.01$ ), but thereafter was similar. Fig. 4 shows the relationship between caloric intake during the first week after birth and the timing of maximum head growth velocity. Infants in groups A and B had similar caloric intakes and also similar gestational ages but showed a difference in the timing of maximum head growth velocity $(P<0.001)$. Infants in groups $\mathbf{B}$ and $\mathbf{C}$ had different caloric intakes $(P<0.01)$ and different gestational ages ( $P<0.001)$ but similar timing of maximum head growth velocity. These observations suggest that the duration of oxygen therapy has more effect on the timing of maximum head growth velocity than the caloric intake in the first week after birth. It may be relevant that $80 \%$ of the infants in group $\mathrm{C}$ were fed with expressed breast milk during the first 2 weeks after birth compared with $11 \%$ of the infants of group B.

The relationship between the timing of maximum growth velocity of OFC and that of body weight is shown in Fig. 5. The timing of the maximum body weight growth was delayed compared with that of head growth, the correlation being significant $(P<0 \cdot 01)$.

Infants in group D with severe respiratory problems but later gestational age (Table 1) had delayed

Table 4 Caloric intake (mean $\pm S D$ ) of infants in groups $A, B$, and $C$ during the first 5 weeks

\begin{tabular}{|c|c|c|c|c|c|}
\hline \multirow[t]{2}{*}{ Group } & \multicolumn{5}{|c|}{ Caloric intake (kcallkg per day) } \\
\hline & 1st week & 2nd week & 3rd week & 4th week & 5th week \\
\hline
\end{tabular}

*Significantly different from groups $\mathbf{A}$ and $\mathbf{B}: \mathbf{P}<0.01$.

Conversion: Traditional units to $S I-$ Calories: $1 \mathrm{kcal} \approx 4 \cdot 18 \mathrm{~kJ}$. 


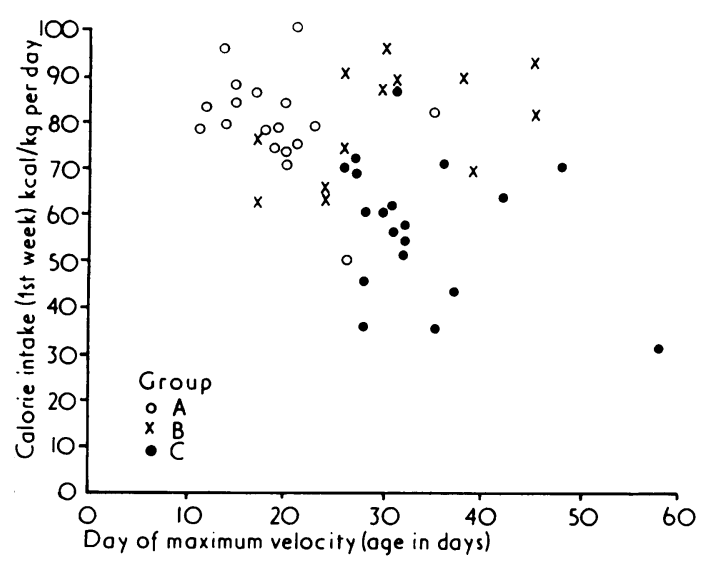

Fig. 4 Timing of maximum velocity of OFC growth plotted against the caloric intake during the first week of life. Conversion: Traditional units to SI-Calories: $1 \mathrm{kcal} \approx 4 \cdot 18 \mathrm{~kJ}$.

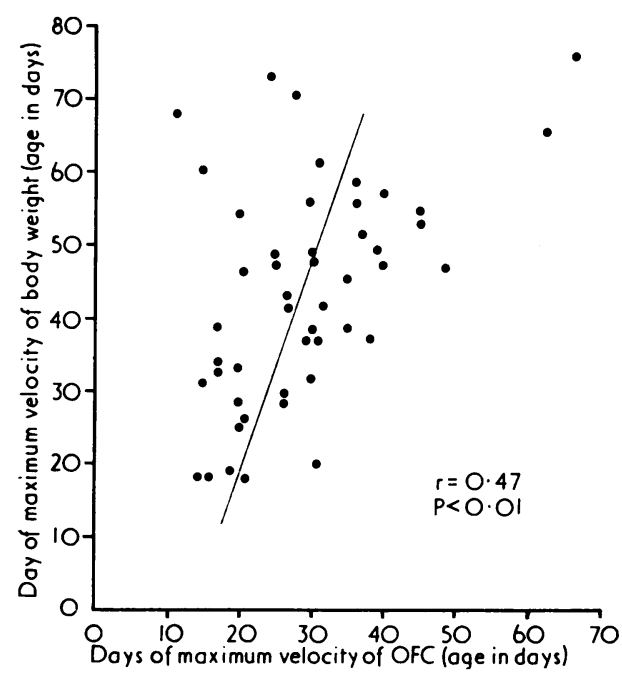

Fig. 5 Relationship between the timing of maximum head growth velocity and maximum body weight velocity.

timing of maximum head growth compared with the infants in group A $(P<0.001)$ who were born at earlier gestation but had at most transient respiratory distress (Table 2).

\section{Discussion}

The method used in this study was found to be simple and accurate for estimating the velocity curve and the timing of maximum velocity of head growth.
In experienced hands the OFC can be reliably measured to within $2 \mathrm{~mm}$ so that one can expect a value of mean velocity with an accuracy of $0.2 \mathrm{~mm} /$ day, taking a pair of OFC values recorded over a gap of 10 days. It is difficult to compare the curves of head growth of individual infants because of the variation in starting OFC measurements and the shape of the curves and their nonlinearity. By looking at the age at which the maximum head growth is achieved one has a single value which is constant for a given gestational age (Table 3). This method can be used to study the effect of illness and treatment on head growth.

Respiratory distress delayed the timing of maximum head growth velocity irrespective of the gestational age. The caloric intake was the same throughout the newborn period in groups $\mathrm{A}$ and $\mathrm{B}$, so that the respiratory illness was the most likely cause of the delay in group B. Infants between 34 and 40 weeks' gestation without respiratory distress may be expected to achieve maximum head growth velocity within 2 weeks of birth (Fujimura and Seryu, 1977). Infants of this gestation suffering from severe respiratory distress showed marked delay in the timing of maximum head growth velocity. Hypoxia may cause this delay. It is difficult now to differentiate the effect of hypoxia on the brain growth from other factors such as the metabolic derangement associated with respiratory distress. It has been reported that in 5-week-old rats there was significant reduction in weight, protein, and DNA content in the cerebellum after exposure to $12 \%$ oxygen during the first week of life (Cheek et al., 1969), though protein restriction did not have any effect on the growth of cerebellum.

There was a positive correlation between the attainment of maximum velocity of body weight on the one hand and OFC on the other. Maximum velocity of head growth was achieved earlier than that of body weight. In individual cases it was difficult to predict the timing of maximum head growth from the timing of maximum body weight growth or vice versa.

The human brain grows most rapidly during the perinatal period and therefore pathological conditions occurring at this time would be more likely to damage the development and function of the brain at this rather than at any other time. The delay of the timing of the maximum head growth velocity may be assumed to be a temporary failure of brain growth. The significance of this delay on future development remains to be examined.

I thank Professor J. P. M. Tizard and Dr. R. O. Robinson for reviewing the manuscript. M. F. was in receipt of a British Council Scholarship. 


\section{References}

Baum, J. D., and Searls, D. (1971). Head shape and size of pre-term low-birthweight infants. Developmental Medicine and Child Neurology, 13, 576-581.

Bray, P. F., Shields, W. D., Wolcott, G. J., and Madsen, J. A. (1969). Occipitofrontal head circumference-an accurate measure of intracranial volume. Journal of Pediatrics, 75, 303-305.

Buda, F. B., Reed, J. C., and Rabe, E. F. (1975). Skull volume in infants. American Journal of Diseases of Children, 129, 1171-1174.

Cheek, D. B., Graystone, J. E., and Rowe, R. D. (1969). Hypoxia and malnutrition in newborn rats: effects of RNA, DNA and protein in tissues. American Journal of Physiology, 217, 642-645.

Davies, P. A., and Davis, J. P. (1970). Very low birthweight and subsequent head growth. Lancet, 2, 1216-1219.

Davies, P. A., Robinson, R. J., Scopes, J. W., Tizard, J. P. M., and Wigglesworth, J. S. (1972). Medical Care of Newborn Babies. Heinemann, London.

Dobbing, J., and Sands, J. (1973). The quantitative growth and development of the human brain. Archives of Disease in Childhood, 48, 757-767.

Fujimura, M., and Seryu, J. (1977). Velocity of head growth during the perinatal period. Archives of Disease in Childhood, 52, 105-112.
Glass, L., Lala, R. V., Jaiswal, V., and Nigam, S. K. (1975). Effect of thermal environment and caloric intake on head growth of low birthweight infants during late neonatal period. Archives of Disease in Childhood, 50, 571-573.

Hey, E. N., and Katz, G. (1970). The optimum thermal environment for naked babies. Archives of Disease in Childhood, 45, 328-334.

O'Neill, E. M. (1961). Normal head growth and the prediction of head size in infantile hydrocephalus. Archives of Disease in Childhood, 36, 241-252.

Sher, P. K., and Brown, S. B. (1975a). A longitudinal study of head growth in pre-term infants. I. Normal rates of head growth. Developmental Medicine and Child Neurology, 17, 705-710.

Sher, P. K., and Brown, S. B. (1975b). A longitudinal study of head growth in pre-term infants. II. Differentiation between 'catch-up' head growth and early infantile hydrocephalus. Developmental Medicine and Child Neuro$\log y, 17,711-718$.

Winick, M., and Rosso, P. (1969). Head circumference and cellular growth of the brain in normal and marasmic children. Journal of Pediatrics, 74, 774-778.

Correspondence to Dr. M. Fujimura, Department of Paediatrics, Yodogawa Christian Hospital, 57 Awaji Honmachi 1-chome, Higashi Yodogawa-ku, Osaka, Japan. 